International Journal of Modern Physics B

(C) World Scientific Publishing Company

\title{
Bose-Einstein condensate in a quartic potential: Static and Dynamic properties
}

\author{
G. K. Chaudhary, Amit K Chattopadhyay, and R. Ramakumar \\ Department of Physics and Astrophysics, University of Delhi, Delhi-11000\%, Delhi, India
}

Received 9 May 2009

\begin{abstract}
In this paper, we present a theoretical study of a Bose-Einstein condensate of interacting bosons in a quartic trap in one, two, and three dimensions. Using Thomas-Fermi approximation, suitably complemented by numerical solutions of the Gross-Pitaevskii equation, we study the ground sate condensate density profiles, the chemical potential, the effects of cross-terms in the quartic potential, temporal evolution of various energy components of the condensate, and width oscillations of the condensate. Results obtained are compared with corresponding results for a bose condensate in a harmonic confinement.
\end{abstract}

Keywords: Bose-Einstein condensation

\section{Introduction}

Since the first experimental observation of Bose-Einstein (BE) condensation $1,2,3$ in bose atom clouds in a harmonic trap, both experimental and theoretical studies of this phenomenon and various properties of the condensate has grown up to a fast expanding field of research 4,5,6,7,8,9,10. While studies of Bose condensation in a harmonic potential is of great interest, investigations of the BE condensation of lattice-bosons and free-bosons in anharmonic potentials, in particular in a quartic potential, have received considerable attention in recent times ${ }^{11,12,13,14,16}$ (see also the note in Ref. ${ }^{17}$ ). All the previous works ${ }^{11,12,13,14}$, except two ${ }^{15,16}$, deals with lattice-bosons in anharmonic confinements. The previous works on free-bosons in power law traps and quartic traps in three and lower dimensions ${ }^{15,16}$ dealt with the calculations of Bose condensation temperature for non-interacting bosons in those environments. In this paper, we consider interacting bosons in a quartic potential. The study of interacting bosons in a quartic potential may have important implications for fields ranging from chaotic dynamics to high energy physics ${ }^{16}$. Further, it would be desirable to have minimum inhomogeneity in the density profiles for clear observation of several phases and transitions possible for interacting boson, fermion, and boson-fermion models ${ }^{9,10}$. While interactions in a many-boson system are expected to broaden the real space distribution of bosons in the confining region, it is not clear how far, from the center, a homogeneous distribution of bosons will exist. We will show, among other results, that the interaction leads to a more or less homogeneous boson distribution in a substantial region (compared 
to the non interacting bosons case) in and around the trap center. It would also be of contemporary interest to investigate the dynamics of the condensate in the specific quartic potential environment and compare it with the usually employed quadratic environment. It may be expected that the experiments would first involve investigations of interacting bosons in a quartic potential before an additional lattice potential is applied. Furthermore, experimental studies of interacting bosons in the simplest anharmonic environment may be of considerable interest in its own right. Within this general setting, it would be useful to make a detailed theoretical study of interacting bosons in a quartic potential. It is the purpose of this paper to present some results of such an investigation using a combination of analytical and numerical methods.

This paper is organized in the following three sections. In Sec 2, the condensate properties are analyzed through a study of the Gross-Pitaevskii equation ${ }^{18,19}$ employing the Thomas-Fermi approximation 20,21 . In Sec 3 we present a comparative study of real space distributions of bosons in condensates of interacting bosons in quartic and harmonic confinements, by numerically solving the Gross-Pitaevskii equation. The time evolution of some properties (various components of energy and width oscillations of the condensate) of the condensate is given in Sec 4, and the conclusions are presented in Sec 5 .

\section{BEC in a quartic trap: Thomas-Fermi approximation}

The ground state properties of a Bose-Einstein condensate (BEC) is well described by a macroscopic wave-function $\psi(\mathbf{r}, t)$, the time evolution of which is governed by the Gross-Pitaeveskii equation 18,19 (GPE). Incorporating the trap potential as well as a repulsive interaction between the atoms forming the condensate, the GPE in three dimensions (3d) is given by

$$
i \hbar \frac{\partial \psi(\mathbf{r}, t)}{\partial t}=-\frac{\hbar^{2}}{2 m} \nabla^{2} \psi(\mathbf{r}, t)+v(\mathbf{r}) \psi(\mathbf{r}, t)+N U_{0}|\psi(\mathbf{r}, t)|^{2} \psi(\mathbf{r}, t),
$$

where $\mathbf{r} \equiv(x, y, z)^{T}, m$ is the atomic mass, $v(\mathbf{r})=\alpha(\mathbf{r} . \mathbf{r})^{2}$ is the quartic trapping potential, $N$ is the total number of atoms forming the condensate, $U_{0}\left(=4 \pi \hbar^{2} a / m\right)$ is the strength of interaction between atoms in the condensed state, and $a(>0)$ is the s-wave scattering length.

Before applying the Thomas-Fermi approximation, we non-dimensionalize Eq. (1) through a set of linear transformations: $\tilde{t}=\omega t, \tilde{\mathbf{r}}=\mathbf{r} / l, \widetilde{\psi}(\mathbf{r})=l^{3 / 2} \psi(\mathbf{r})$. After dropping the wiggles on the symbols, we obtain

$$
i \frac{\partial \psi(\mathbf{r}, t)}{\partial t}=-\frac{1}{2} \nabla^{2} \psi(\mathbf{r}, t)+\gamma(\mathbf{r} . \mathbf{r})^{2} \psi(\mathbf{r}, t)+\lambda N|\psi(\mathbf{r}, t)|^{2} \psi(\mathbf{r}, t),
$$

where

$$
l=\sqrt{\frac{\hbar}{m \omega}}, \quad \lambda=\frac{4 \pi a}{l}, \quad \gamma=\frac{\alpha \hbar}{m^{2} \omega^{3}} .
$$


In the absence of the non-linear interaction term, the GPE is reduced to a Schrödinger equation (with a quartic potential) whose approximate eigenvalues can be obtained through perturbation theory or WKB approximation 22 . Expressing $\alpha$ in units of $m^{2} \omega^{3} / \hbar$, we perform dimensional reductions 23 to obtain corresponding GPEs in lower dimensions. In general, the GPEs for $d=1,2$, and 3 can be summarized in a single equation as

$$
i \frac{\partial \psi(\mathbf{r}, t)}{\partial t}=-\frac{1}{2} \nabla^{2} \psi(\mathbf{r}, t)+(\mathbf{r} . \mathbf{r})^{2} \psi(\mathbf{r}, t)+k_{d}|\psi(\mathbf{r}, t)|^{2} \psi(\mathbf{r}, t),
$$

where

$$
k_{d}=\alpha_{d} \lambda_{d} N, \quad \lambda_{d}=\frac{4 \pi a}{l^{d-2}} .
$$

Here $\alpha_{2}$ and $\alpha_{1}$ are scaling factors introduced to write GPE in $2 \mathrm{~d}$ and $1 \mathrm{~d}$, respectively. In order to find a stationary solution of Eq. (4), we do a separation of variables $\psi(\mathbf{r}, t)=\psi(\mathbf{r}) \times \exp [-i(\mu /(\hbar \omega)) t]$, where $\mu$ is the chemical potential. Starting from Eq. (4), we obtain

$$
-\frac{1}{2} \nabla^{2} \psi(r)+r^{4} \psi(r)+k_{d}|\psi(r)|^{2} \psi(r)=\frac{\mu}{\hbar \omega} \psi(r) .
$$

In the preceding equation $r^{4} \equiv x^{4}+y^{4}+z^{4}$. For simplicity we have neglected the cross terms from the potential (see, however, Sec. III-C where these terms are shown to have only minor effects on the properties we study). To analytically solve for the ground state of the stationary state GPE, as given in Eq. (6), we make use of the Thomas-Fermi approximation 4,20,21,24. For sufficiently large number of atoms, the kinetic energy is very small compared to the potential and the interaction energies. So, a good approximation to the ground state is obtained by solving the GPE without the kinetic energy term. On using this approximation, the GPE becomes

$$
r^{4} \psi(r)+k_{d}|\psi(r)|^{2} \psi(r)=\frac{\mu}{\hbar \omega} \psi(r),
$$

The relevant solutions of the preceding cubic equation are

$$
\psi(r)= \pm \sqrt{\frac{1}{k_{d}}\left(\frac{\mu}{\hbar \omega}-r^{4}\right)} .
$$

Since $\psi(r)$ vanishes for $\frac{\mu}{\hbar \omega} \leq r^{4}$, the Thomas-Fermi radius $r_{\mathrm{TF}}$ is obtained as

$$
r_{\mathrm{TF}}=\left(\frac{\mu}{\hbar \omega}\right)^{1 / 4}
$$

in which the chemical potential $\mu_{\mathrm{TF}}$ is be determined from the normalization condition

$$
c_{d} \int_{0}^{r_{\mathrm{TF}}}|\psi(r)|^{2} r^{d-1} d r=1,
$$

where $c_{d}=2,2 \pi$, and $4 \pi$ for $d=1,2$, and 3 , respectively. Then, the chemical potential is found to be

$$
\mu_{\mathrm{TF}}^{d}=\hbar \omega\left[\frac{d(d+4)}{4 c_{d}} k_{d}\right]^{\frac{4}{d+4}}
$$


So, for the $3 \mathrm{~d}$ case, the chemical potential varies as $N^{\frac{4}{7}}$ for the quartic trap. In comparison, we note that it varies as $N^{\frac{2}{5}}$ for bosons in a harmonic trap ${ }^{23,26}$ (for which $v(\mathbf{r}) \propto \mathbf{r . r})$. It may be noted that the $N$ dependence of the chemical potential becomes more dominant as the dimensionality decreases.

The scaling factors $\left(\alpha_{2}\right.$ and $\left.\alpha_{1}\right)$ were introduced in $2 \mathrm{~d}$ and $1 \mathrm{~d}$ GPE to obtain GPEs in $2 \mathrm{~d}$ and $1 \mathrm{~d}$. They also ensure that the size of the condensate remains the same in all the three dimensions. To find $\alpha_{2}$ and $\alpha_{1}$, we calculate $\mu_{T F}$ in $2 \mathrm{~d}$ and $1 \mathrm{~d}$ and equate it to the chemical potential obtained in $3 \mathrm{~d}^{23}$. Using this procedure, we obtain

$$
\begin{gathered}
\alpha_{3}=1 \\
\alpha_{2}=\frac{1}{6}\left(\frac{21}{4}\right)^{\frac{6}{7}} N^{\frac{-1}{7}}\left(a l^{6}\right)^{-\frac{1}{7}}
\end{gathered}
$$

and

$$
\alpha_{1}=\frac{2}{5 \pi}\left(\frac{21}{4}\right)^{\frac{5}{7}} N^{\frac{-2}{7}}\left(a^{2} l^{12}\right)^{-\frac{1}{7}}
$$

\section{Spatial distribution of bosons in the condensate}

\subsection{Condensate density profiles in 1d}

In this section, we present results on the spatial distribution of bosons in the condensate in $1 \mathrm{~d}$ by numerically solving the GPE. We solve the GPE by using the finite difference Crank-Nicholson (FDCN) method $^{23,25}$. In this method for the GPE given by

$$
i \frac{\partial \psi(x, t)}{\partial t}=H \psi(x, t)
$$

the solution is advanced in small time steps $\delta t$ as given below

$$
\psi(x, t+\delta t)=\frac{1-i \frac{\delta t}{2} H}{1+i \frac{\delta t}{2} H} \psi(x, t) .
$$

The ground state solution is found by the imaginary time propagation method 23 . In the imaginary time propagation method, we replace $\delta t$ by $-i \delta t$ and propagate a initial trial wave function using the above mentioned propagation scheme. After suitable normalization at each time step the wave-function converges to the ground state. We have taken the space step as $\delta x=0.1$ and the time step as $\delta t=0.001$. For illustrative purpose, we have used a set of parameters for ${ }^{87} \mathrm{Rb}: m=1.44 \times$ $10^{-25} \mathrm{Kg}, a=5.1 \times 10^{-9} \mathrm{~m}, \nu(=\omega / 2 \pi)=24 \mathrm{~Hz}$.

In Fig. 1, we show the condensate density profiles for systems with 10 to $10^{6}$ bosons in a quartic trap. Further, this figure also contains results of the TF approximation for the same numbers of bosons in a quartic trap. Furthermore, we have also shown the results for bosons in a harmonic potential. We find that the TF approximation results are in quantitative agreement with the numerical solutions 
of GPE when the number of bosons is large. On increasing the number of atoms, the width of the ground state increases, which is similar to the case of bosons in a harmonic potential ${ }^{23,26}$, except that the spread of condensate is larger for a harmonic potential. We also notice that the ground state density profiles for the quartic trap becomes relatively flat with increasing number of bosons in the quartic trap. Next, we fix the number of atoms to $10^{4}$ in a quartic trap and investigate the boson distribution in the ground state for different interaction strengths. The results are displayed in the left panel of Fig. 2. We find that the ground state boson distribution is more spread out with increasing interaction strength. It may be argued that the redistribution of bosons from the center to other parts of the condensate is due to the peak in the non-interacting distribution in the central region since the $U=0$ wave-function is peaked at the center and is symmetric around the center. But, for the interacting bosons case, the interaction term is to be calculated in a self-consistent manner including the effect of the interaction. Hence, it is not obvious that interacting bosons distribution will have the profile shown in Fig. 2. It is the result of a combined effect of interaction and self-consistent changes in the wavefunction. Thus, we see that the interacting bosons, compared to the non-interacting bosons, have a density profile which is more or less uniform in the central region in the case of a quartic confining potential. In Fig. 2, we have also exhibited the ground state chemical potential for different values of the interaction parameter $k_{1}$. The chemical potential for a condensate in a quartic trap is larger than that in harmonic trap, the difference becoming more significant for higher values of $k_{1}$.

\subsection{Condensate density profiles in $2 d$ and $3 d$}

We have solved the GPE in higher dimensions by using the split-step FDCN $\operatorname{method}^{27}$. In this method, in $3 \mathrm{~d}$, the Hamiltonian $H$ is split into different nonderivative and derivative parts $H_{1}, H_{2}, H_{3}$, and $H_{4}$, where

$$
\begin{gathered}
H_{1}=v(\mathbf{r})+k_{3}|\psi(\mathbf{r}, t)|^{2} \\
H_{2}=-\frac{1}{2} \frac{\partial^{2}}{\partial x^{2}}, H_{3}=-\frac{1}{2} \frac{\partial^{2}}{\partial y^{2}}, H_{4}=-\frac{1}{2} \frac{\partial^{2}}{\partial z^{2}} .
\end{gathered}
$$

The time evolution is performed in the following steps. Let $\psi^{n}$ be the wave-function at time $t_{n}$. This wave-function is advanced first over a time step $\delta t$ at $t_{n}$ to produce an intermediate solution $\psi^{n+1 / 4}$ from $\psi^{n}$ via

$$
\psi^{n+1 / 4}=e^{-i \delta t H_{1}} \psi^{n} .
$$

Next the time propagation is performed via the following semi-implicit CrankNicholson scheme:

$$
\psi^{n+2 / 4}=\frac{1-i \frac{\delta t}{2} H_{2}}{1+i \frac{\delta t}{2} H_{2}} \psi^{n+1 / 4}
$$




$$
\psi^{n+3 / 4}=\frac{1-i \frac{\delta t}{2} H_{3}}{1+i \frac{\delta t}{2} H_{3}} \psi^{n+2 / 4},
$$

and

$$
\psi^{n+1}=\frac{1-i \frac{\delta t}{2} H_{4}}{1+i \frac{\delta t}{2} H_{4}} \psi^{n+3 / 4} .
$$

An analogous set of equations hold in $2 \mathrm{~d}$. In the calculations, we have used space steps of 0.1 (for $\delta x$ and $\delta y$ in $2 \mathrm{~d}$ and $\delta x, \delta y$, and $\delta z$ in $3 \mathrm{~d}$ ) and the time step $\delta t=0.001$. To calculate the ground state, again we use imaginary time propagation method by replacing $\delta t$ with $-i \delta t$. We start with an initial trial wave-function which is propagated via the above mentioned scheme. After suitable normalization, at each time step, the wave-function converges to the ground state.

In Fig. 3, the ground state density profiles of the condensate are shown for different numbers of atoms ranging from $10^{3}$ to $10^{6}$. On increasing the number of atoms, the width of the ground state increases, which is similar to that in $1 \mathrm{~d}$. On comparing the ground states for harmonic and quartic traps as shown in Fig. 4, we found that the ground state density profiles are flat in the central region for the quartic trap compared to the harmonic trap. Similar results are obtained for the $3 \mathrm{~d}$ case as well. Clearly, the quartic confinement is more advantageous to the quadratic one if one is interested in investigations of the properties of a bose condensate with a minimal influence of the confining potential. We may note here that, in an earlier work $^{12}$, we have shown that the finite temperature properties of lattice bosons are closer to the pure lattice case if one uses quartic rather than a quadratic confining potential. The effect of changing interaction strength on the ground state density profiles in a quartic trap are displayed in Figs. 5 and 6 for $2 d$ and 3d, respectively. It is clear that the bosons gain energy by spreading into larger area in the traps with increasing strength of the interaction. In Fig. 7, the ground state chemical potential is plotted for different values of the interaction parameter $k_{d}$ for $d=2$ and 3 . The chemical potentials of the condensate in a quartic traps are higher than those in harmonic traps, the difference becoming more significant at higher values of $k_{d}$.

\subsection{Effects of cross terms}

In the preceding section, we have presented results of our calculations neglecting the cross terms in the full quartic potential $\left(V(\mathbf{r})=\alpha(\mathbf{r} . \mathbf{r})^{2}\right)$. In this section, we investigate the effects of the previously neglected cross terms. The contribution of the cross terms is in cross-coupling potentials across different dimensions. One may expect that the inclusion of these cross-terms may lead to complex inhomogeneous density profiles. But, on performing the calculations for the quartic trap, we find that the effects of cross-terms are rather minor. They lead to small increases in the chemical potential and the number of bosons in the central region of the trap as shown in Fig. 8 for 2d. We do not find any significant inhomogeneities in the ground state condensate profiles generated by the cross-terms. We have observed 
(figures not shown) similar effects in $3 \mathrm{~d}$ as well. It may be noted here that cross terms lead to a $10 \%$ increase of the bose condensation temperature $\left(T_{c}\right)$ in $2 \mathrm{~d}$, and a $20 \%$ increase in $3 \mathrm{~d}^{16}$.

\section{Time evolution of the condensate}

In this section, we study the time evolution of the condensate after removal of the trapping potential and width oscillations of the condensate. To theoretically study the expansion of condensate, we time evolve the GPE after removing the potential term, taking the stationary ground state as the initial state function at $t=0$. To time evolve the GPE we use the FDCN scheme given by Eqs. 15-16 in $1 \mathrm{~d}$ and split-step FDCN scheme as given in Eqs. 17-22 in higher dimensions. Here the propagation is done in real time with time-step $\delta t=0.0001$ and space steps of 0.1 (for $\delta x, \delta y$, and $\delta z$ ). The different energy components we calculate are given by 4,28 :

$$
\begin{aligned}
& E_{\mathrm{kin}}(t)=-\frac{1}{2} \int \psi^{*}(r, t) \nabla^{2} \psi(r, t) d r \\
& E_{\mathrm{pot}}(t)=\int \psi^{*}(r, t) v(r) \psi(r, t) d r, \\
& E_{\mathrm{int}}(t)=\frac{1}{2} \int \psi^{*}(r, t) k_{d}|\psi(r, t)|^{2} \psi(r, t) d r .
\end{aligned}
$$

And, the the chemical potential is given by ${ }^{4,28}$ :

$$
\mu(t)=\int\left(\frac{1}{2}|\nabla \psi(r, t)|^{2}+v(r)|\psi(r, t)|^{2}+k_{d}|\psi(r, t)|^{4}\right) d r
$$

The variation of various energy components and the chemical potential, for bosons in quartic and harmonic traps, with time is shown in Fig. 9 for $1 \mathrm{~d}$ and $2 \mathrm{~d}$. Similar results (not shown) are observed in 3d. As shown in these figures, the chemical potential and the interaction energy decreases on time evolution, but there is an increase in kinetic energy term. Most of the energy before the expansion $(t<0)$ is contained in the mean-field and the potential energy of the bosons. At $t=0$, the confining potential is removed and the corresponding energy term from then on is zero. During the expansion, the chemical potential decreases due to decrease in the mean field. Further, the increase in kinetic energy during the expansion is due to transfer from the mean-field. We have also shown in Fig. 9, the release energy $\left(E_{\text {rel }}=E_{\text {kin }}+E_{\text {int }}\right)$ which is a a time independent quantity.

The time evolution of the width of the condensate is displayed in Fig. 10 for 1d, 2d, and 3d. The condensate widths along three axes $\mathrm{x}, \mathrm{y}$, and $\mathrm{z}$ are defined as $\Delta x=$ $\sqrt{\left\langle(x-\langle x\rangle)^{2}\right\rangle}, \Delta y=\sqrt{\left\langle(y-\langle y\rangle)^{2}\right\rangle}$, and $\Delta z=\sqrt{\left\langle(z-\langle z\rangle)^{2}\right\rangle}$, where brackets $<>$ represent the expectation value at a particular instant during the time evolution. We assume that at $t=0$, the condensate is in a non-interacting ground state. Once the interaction is switched on at $t=0$, it produces oscillations in the condensate. The Fig. 10 show that the frequencies of oscillations are greater in the quartic potential case than in the harmonic case ${ }^{29}$. For isotropic quartic 
potential, amplitude and frequency of oscillations in $\mathrm{x}, \mathrm{y}$, and $\mathrm{z}$ directions are the same.

\section{Conclusions}

In this paper, we studied the static and dynamic properties of a $\mathrm{BE}$ condensate in a quartic trap in one, two, and three dimensions. The analysis was done using both analytical and complementary numerical techniques. The analytical solutions were obtained using the Thomas-Fermi approximation. The solutions obtained were then compared with the results obtained by numerically solving the GPE. We compared the ground state density profiles of the condensate in a quartic trap for different strengths of interaction by changing the scattering length. It was found that the Thomas-Fermi approximation results were in remarkably good agreement with the results obtained from the GPE for a large number of atoms in the condensate in a quartic confining potential. The results were also compared with corresponding results for a bose condensate in a harmonic confining potential. We find that the condensate density profiles are relatively flat in the central region of the quartic potential compared to the case of a condensate in a harmonic potential. An implication of this result is that for investigating properties of a bose condensate with minimal influence of the overall confining potential, the quartic potential is more preferable compared to a quadratic potential. We also find that increasing the number of atoms in the condensate or increasing the interactions spreads the condensate in the confining region. Our calculations of the chemical potential in the ground state show that it is larger for a condensate in a quartic trap compared to that in a harmonic trap. The preceding conclusions were arrived at without including the cross-terms in a general quartic potential. On including the cross-terms, we find that their effects lead to increases in the chemical potentials and condensate density in the central region of the trap. These changes were found to be rather minor. From our results for the time evolution of the various energy components of an expanding condensate (after removing the confining potentials), we find that the chemical potential and the potential energy decrease during the expansion while the kinetic energy increases. It was also noticed that changes in the case of quartic potential are larger than the case of a harmonic potential. The condensate width oscillations produced by switching on of the interaction, starting with a condensate of non-interacting bosons, were also studied. It was found that the width oscillations of a condensate in a quartic trap have higher frequency than that in a harmonic trap. 


\section{Acknowledgment}

Gopesh Kumar Chaudhary thanks UGC, Government of India, for financial support during this work.

1. M. H. Anderson, J. R. Ensher, M. R. Matthews, C. E. Weiman, and E. A. Cornell, Science 269, 198 (1995).

2. K. B. Davis, M. -O. Mewes, M. R. Andrews, N. J. van Druten, D. S. Durfee, D. M. Kurn, and W. Ketterle, Phys. Rev. Lett. 75, 3969 (1995).

3. C. C. Bradley, C. A. Sackett, J. J. Tollett, and R. G. Hulet, Phys. Rev. Lett. 75, 1687 (1995).

4. F. Dalfovo, S. Giorgini, L. P. Pitaevskii, and S. Stringari, Rev. Mod. Phys. 71, 463 (1999).

5. A. J. Leggett, Rev. Mod. Phys. 73, 307 (2001).

6. J. R. Anglin and W. Ketterle, Nature 416, 211 (2002).

7. C. J. Pethick and H. Smith, Bose-Einstein condensation in dilute gases (Cambridge University Press, Cambridge UK, 2002).

8. I. Bloch, Nature Phys. 1, 23 (2005).

9. I. Bloch, J. Dalibard, and W. Zwerger, Rev. Mod. Phys. 80, 885 (2008).

10. V. I. Yukalov, Laser Physics 19, 1 (2009).

11. O. Gygi, H. G. Katzgraber, M. Troyer, S. Wessel, and G. G. Batrouni, Phys. Rev. A 73, 063606 (2006).

12. R. Ramakumar and A. N. Das, Eur. Phys. J. D 47, 203 (2008).

13. E. Lundh, Eur. Phys. J. D 46, 517 (2008).

14. P. N. Ma, K. Y. Yang, L. Pollet, J. V. Porto, M. Troyer, and F. C. Zhang, arXiv:0803.0546v1 [cond-mat.other].

15. V. Bagnato and D. Kleppner, Phys. Rev. A 44, 7439 (1991).

16. S. Gautam and D. Angom, Eur. Phys. J. D 46, 151 (2008).

17. We note here that a large body of work has been done for bosons in mixed harmonic and quartic traps, starting with the suggestion of Fetter that large vortices can be stabilized in fast rotating bose condensates in a harmonic trap by adding a quartic part to the confining potential [see, for example, A. L. Fetter, Phys. Rev. A 64, 063608 (2001); V. Bretin, S. Stock, Y. Seurin, and J. Dalibard, Phys. Rev. Lett. 92, 050403 (2004), and references there in].

18. E. P. Gross, Nuovo Cimento 20, 454 (1961).

19. L. P. Pitaevskii, Zh. Eksp. Teor. Fiz. 40, 646 (1961) [Sov. Phys. JETP 13, 451 (1961)].

20. L. H. Thomas, Math. Proc. Cambridge Philos. Soc. 23, 542 (1927).

21. E. Fermi, Z. Phys. 48, 73 (1928).

22. P. M. Mathews, M. Seetharaman, and S. Raghavan, J. Phys. A 15, 103 (1982).

23. J. E. Williams, The preparation of topological modes in a strongly-coupled twocomponent Bose-Einstien condensate (Ph. D. Thesis, University of Colorado, Boulder, 1999).

24. G. Baym and C. J. Pethick, Phys. Rev. Lett. 76, 6 (1996).

25. S. K. Adhikari and P. Muruganandam, J. Phys. B: At. Mol. Opt. Phys. 35, 2831 (2002).

26. W. Bao and W. Tang, J. Comput. Phys. 187, 230 (2003).

27. P. Muruganandam and S. K. Adhikari, J. Phys. B: At. Mol. Opt. Phys. 36, 2501 (2003).

28. M. J. Holland, D. S. Jin, M. L. Chiofalo, and J. Cooper, Phys. Rev. Lett. 78, 3801 (1997).

29. W. Bao, D. Jaksch, and A. Markowich, J. Comput. Phys. 187, 318 (2003). 

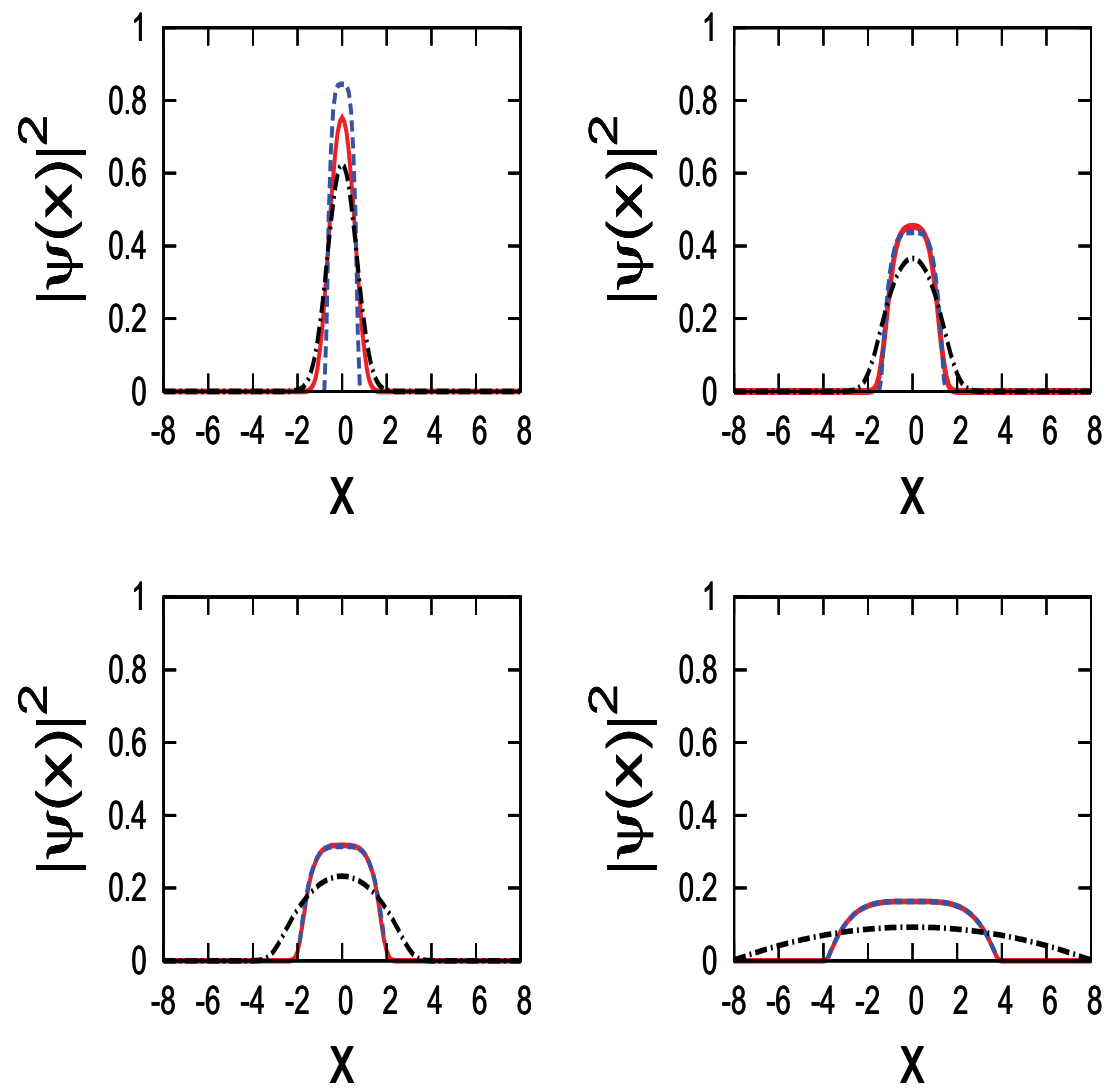

Fig. 1. The density profiles of the condensate ground state in $1 \mathrm{~d}$ for various values of N: 10 (top left panel), $10^{3}$ (top right panel), $10^{4}$ (bottom left panel), $10^{6}$ (bottom right panel). The results are for: the quartic trap (solid lines), TF results for the quartic trap (dotted lines), and for the harmonic trap (dash-dot lines). In this and other figures $\mathrm{x}$ is in units of $l$. The value of $\lambda_{1}$ is $1.41 \times 10^{-13} \mathrm{~m}^{2}$. 

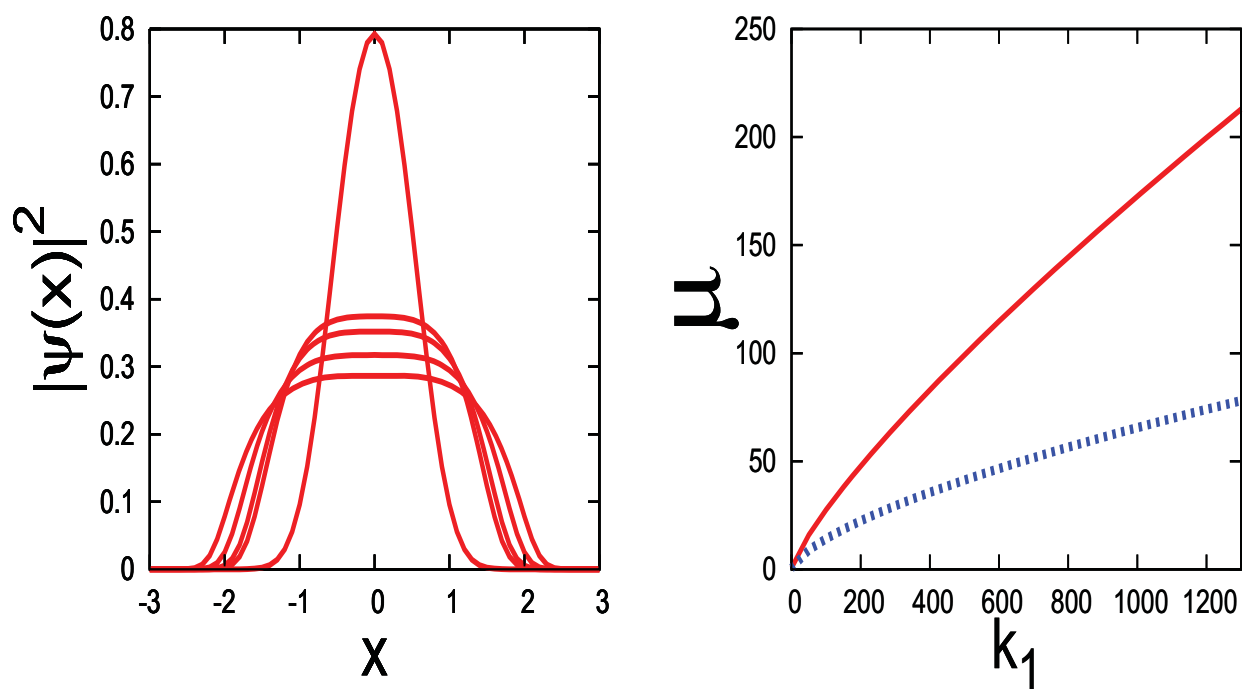

Fig. 2. Left panel: The density profiles of the condensate ground state of $10^{4}$ bosons in a $1 d$ quartic potential for different values of the interaction parameter $k_{1}$. The curves are for (from top to bottom): $k_{1}=0,22.52,30.07,49.34,80.95$. Right Panel: Ground state chemical potential $\mu$ for various values of interaction $k_{1}$ in $1 \mathrm{~d}$ for bosons in a quartic potential (solid line) and in a harmonic potential (dotted line). The $\mu$ is in units of $\hbar \omega$ and $x$ is in units of $l$.
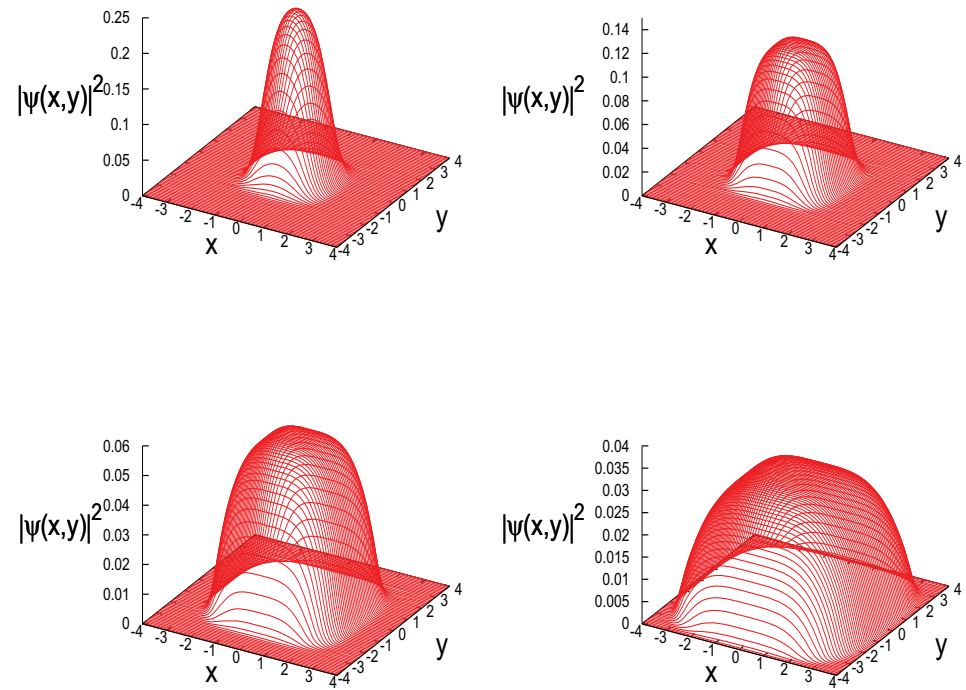

Fig. 3. The density profiles of the condensate ground state in 2 d quartic trap for: $N=10^{3}$ (top left), $N=10^{4}$ (top right), $N=10^{5}$ (bottom left), and $N=10^{6}$ (bottom right). The value of $\lambda_{2}$ is $6.408 \times 10^{-8} \mathrm{~m}$. The $x$ and $y$ are in units of $l$. 

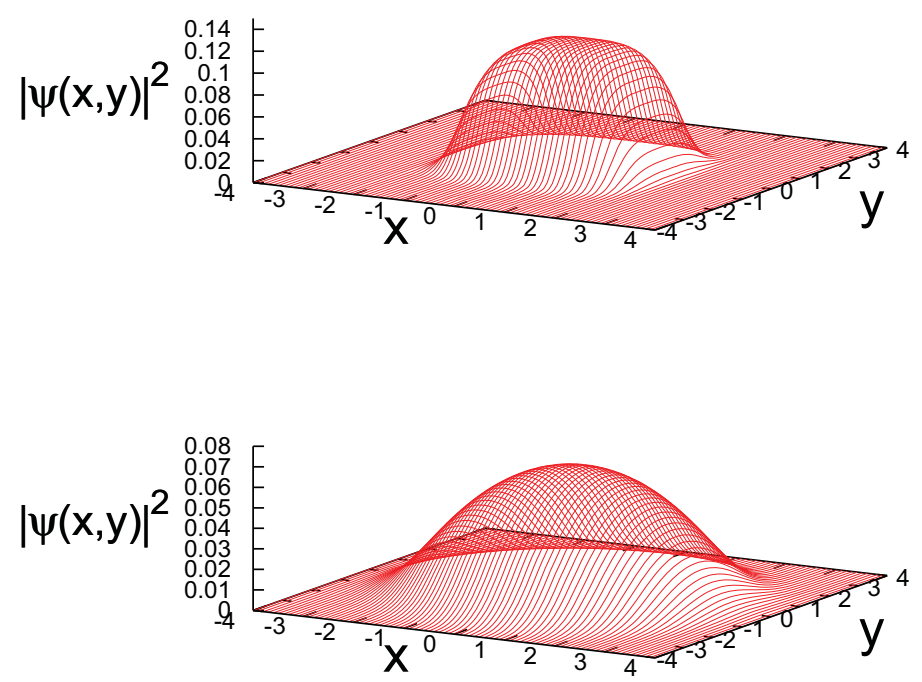

Fig. 4. The density profiles of the condensate ground state in $2 \mathrm{~d}$ for $\mathrm{N}=10^{4}$ for the quartic potential (top) and for the harmonic potential (bottom). The value of $\lambda_{2}$ is $6.408 \times 10^{-8} \mathrm{~m}$. The $x$ and $y$ are in units of $l$.
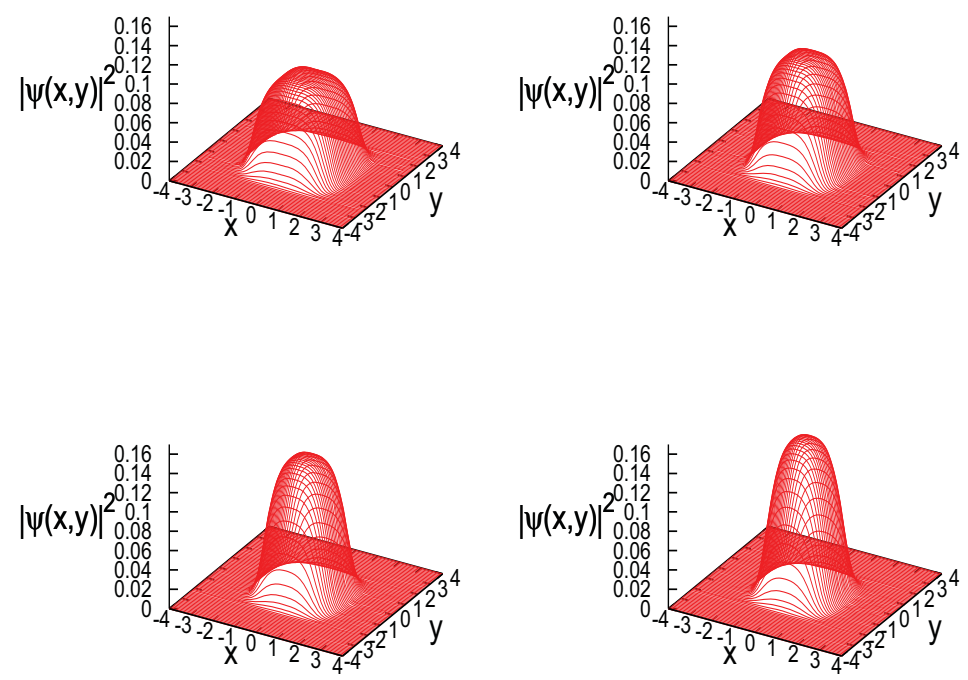

Fig. 5. The density profiles of the condensate ground state in $2 \mathrm{~d}$ for the quartic potential for different values of the interaction parameter $k_{2}$. The figures are for $N=10^{4}$ and for: $k_{2}=232.25$ (top left), 128.21 (top right), and 70.78 (bottom left), 50.00 (bottom right). The $x$ and $y$ are in units of $l$. 

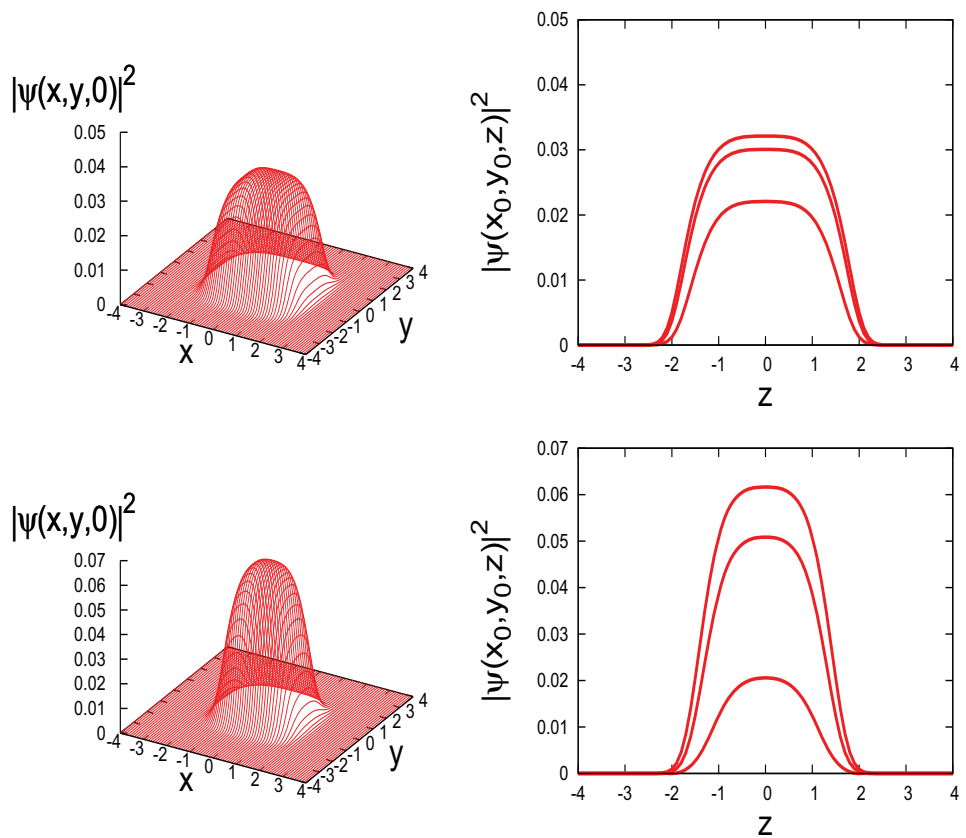

Fig. 6. The density profiles of the condensate ground state in $3 \mathrm{~d}$ for a quartic potential for different values of the interaction parameter $k_{3}$. These figures are for $N=10^{4}$ and for: $k_{3}=$ 581.79 (top), 145.44 (bottom). The values of $\left(x_{0}, y_{0}\right)$ are: $(0.1,0.1)$ (top), (0.1,1.5) (middle), and $(0.1,2.0)$ (bottom). The $x, y$, and $z$ are in units of $l$.
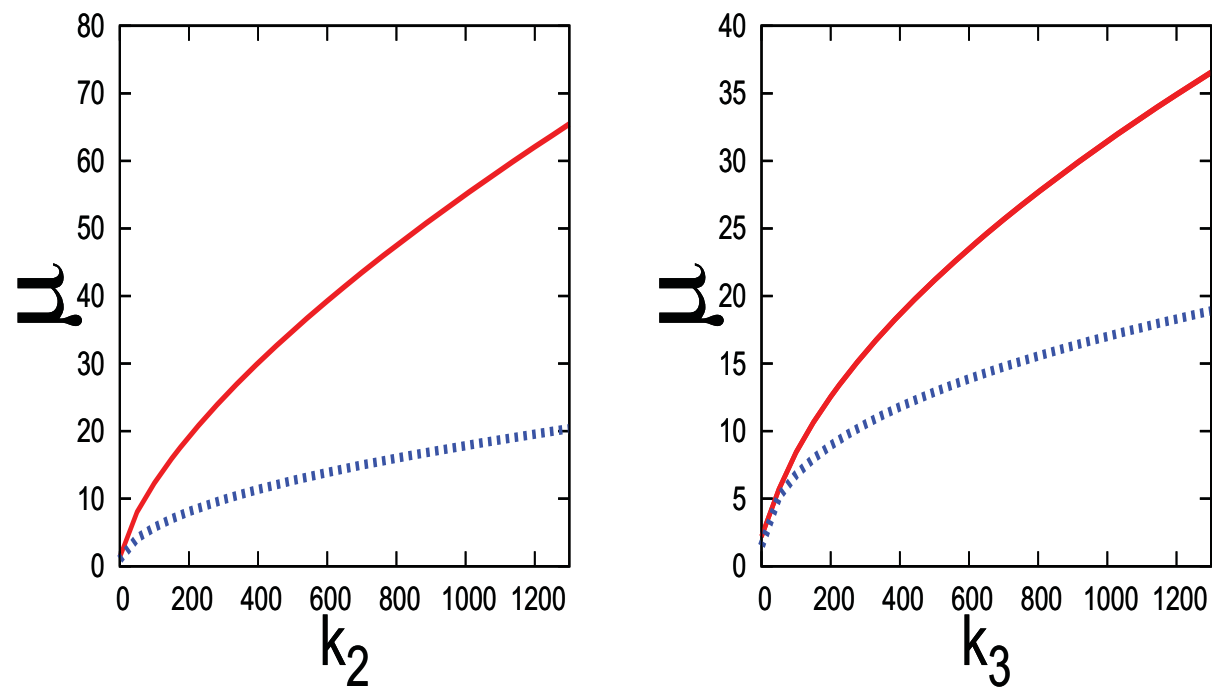

Fig. 7. A comparison of chemical potentials with interaction strengths for bosons in $2 \mathrm{~d}$ (left panel) and in $3 \mathrm{~d}$ (right panel) quartic (solid lines) and harmonic potentials (dotted lines). The $\mu$ is in units of $\hbar \omega$. 

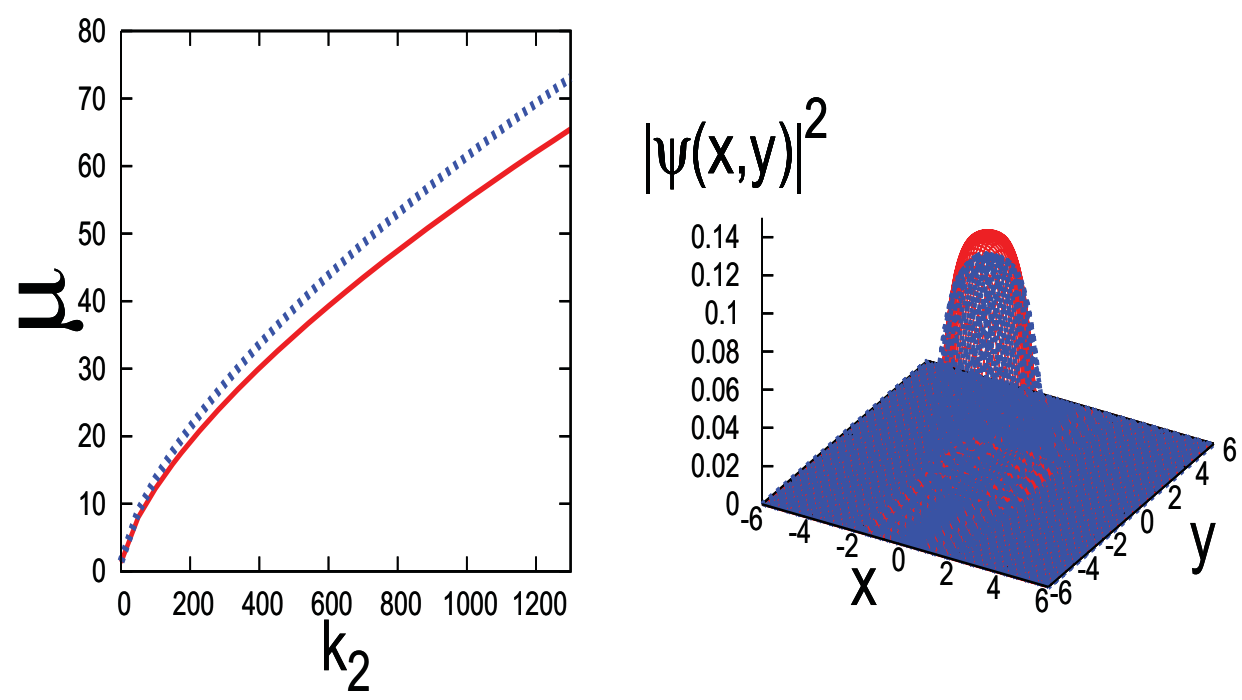

Fig. 8. Left panel: The variation of ground state chemical potential with interaction strength $k_{2}$, in a $2 \mathrm{~d}$ quartic potential, including cross-terms (dotted line) and without cross-terms (solid line). Right panel: The density profiles of the condensate ground state in a $2 \mathrm{~d}$ quartic potential for quartic potential with cross terms (solid line) and without cross terms (dotted line). The results shown are for $N=10^{4}$ and $\lambda_{2}=6.408 \times 10^{-8} \mathrm{~m}$. The $\mu$ is in units of $\hbar \omega$ and the $x$ and $y$ are in units of $l$.
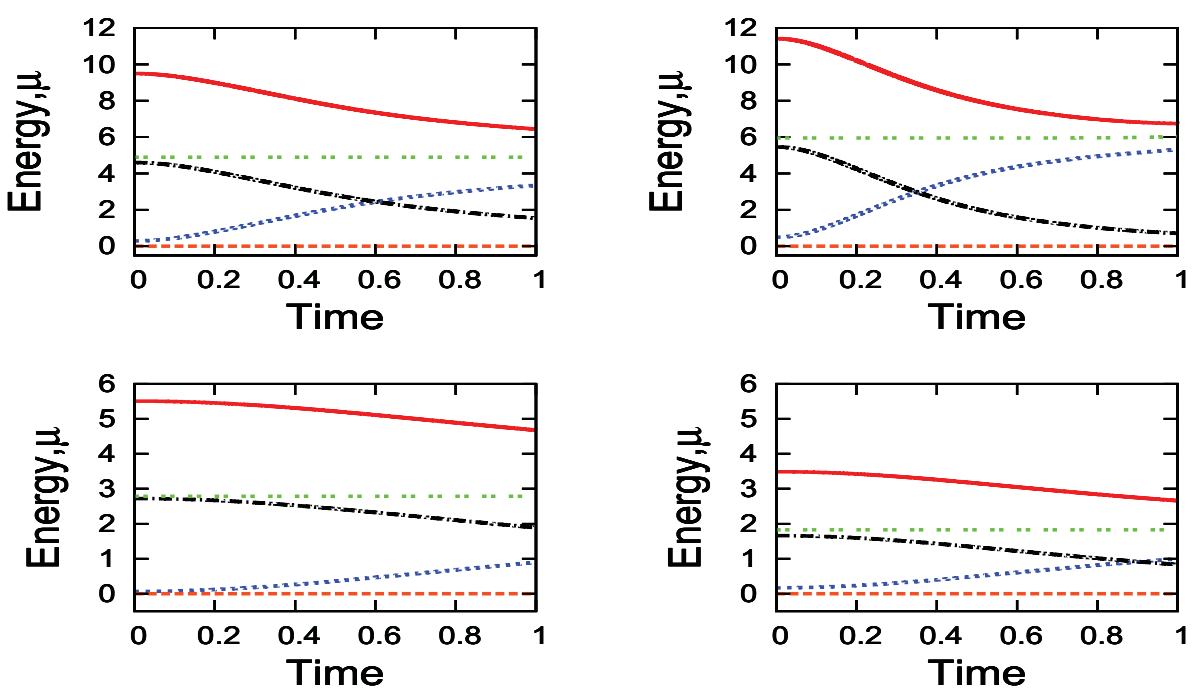

Fig. 9. The evolution of chemical potential $(\mu)$ and energy components with time in 1d (left panels) and $2 \mathrm{~d}$ (right panels). The chemical potential (solid lines), the release energy (double-dot lines), the interaction energy (dash-dot lines), the kinetic energy (dotted lines), the potential energy (dashed lines). The top panels are for quartic potential and the bottom panels are for a harmonic potential. The results shown are for $N=10^{4}, \lambda_{1}=1.41 \times 10^{-13} \mathrm{~m}^{2}$, and $\lambda_{2}=6.408 \times 10^{-8} \mathrm{~m}$. All the energies and $\mu$ are in units of $\hbar \omega$ and the time is in units of $\omega$. 

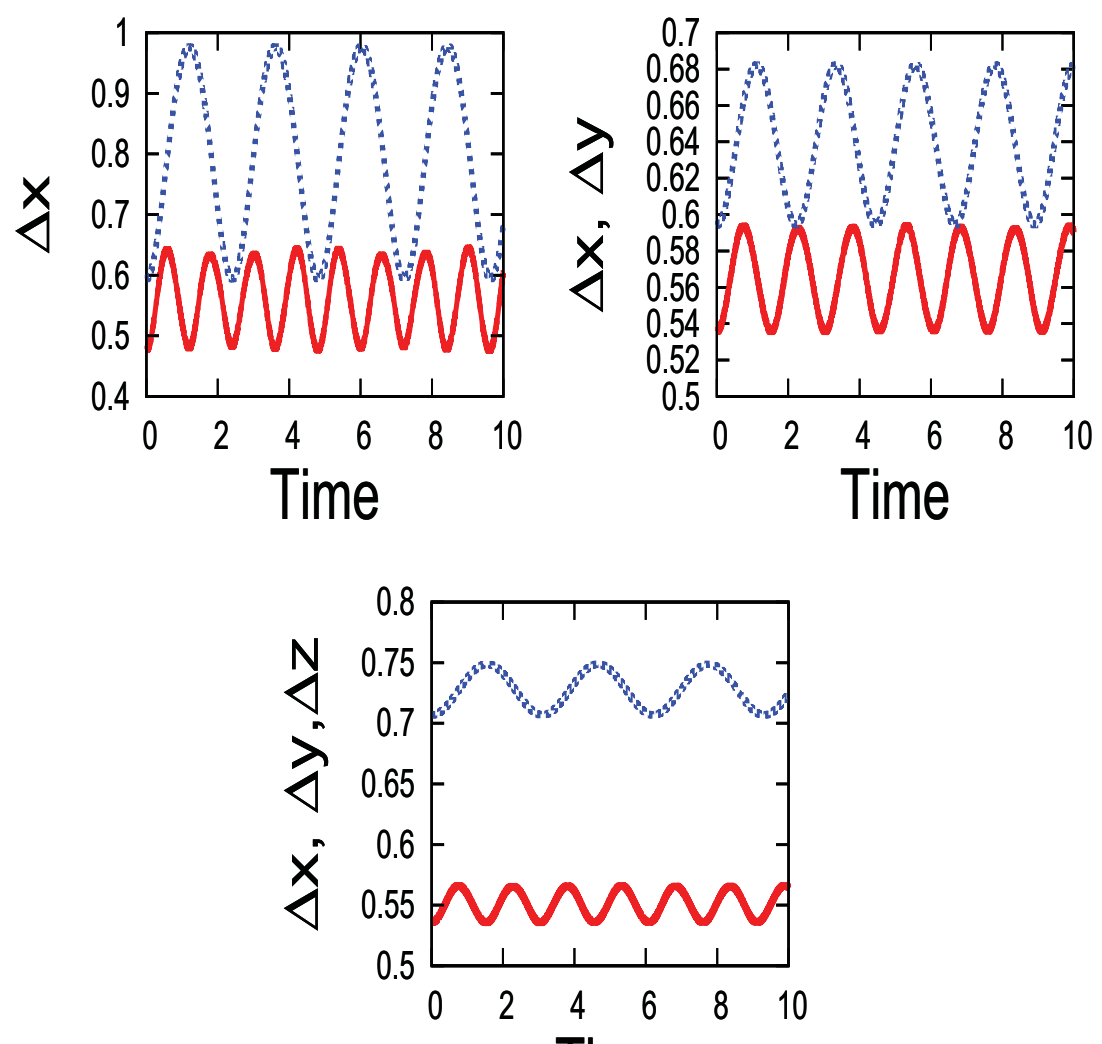

Time

Fig. 10. Condensate widths $(\Delta x, \Delta y, \Delta z)$ as a function of time in: $1 \mathrm{~d}$ (top left), $2 \mathrm{~d}$ (top right), and $3 \mathrm{~d}$ (bottom). The values of $k_{1}, k_{2}$, and $k_{3}$ are 2.0. The solid lines are for the quartic potential and the dotted lines for harmonic potential. The time is in units of $\omega$. 